Publications of the Astronomical Society of the Pacific, 119: 1133-1144, 2007 October

(C) 2007. The Astronomical Society of the Pacific. All rights reserved. Printed in U.S.A.

\title{
Spectral Mapping Reconstruction of Extended Sources
}

\author{
J. D. T. Smith, ${ }^{1}$ L. Armus, ${ }^{2}$ D. A. Dale,${ }^{3}$ H. Roussel,${ }^{4}$ K. Sheth,${ }^{2}$ B. A. Buckalew,${ }^{5}$ T. H. Jarrett,${ }^{5}$ G. Helou, ${ }^{5}$ And \\ R. C. KenNicutt, JR. ${ }^{1,6}$ \\ Received 2007 June 5; accepted 2007 August 21; published 2007 October 25
}

\begin{abstract}
Three-dimensional spectroscopy of extended sources is typically performed with dedicated integral field spectrographs. We describe a method of reconstructing full spectral cubes, with two spatial and one spectral dimension, from rastered spectral mapping observations employing a single slit in a traditional slit spectrograph. When the background and image characteristics are stable, as is often achieved in space, the use of traditional long slits for integral field spectroscopy can substantially reduce instrument complexity over dedicated integral field designs, without loss of mapping efficiency-particularly compelling when a long-slit mode for single unresolved source follow-up is separately required. We detail a custom flux-conserving cube reconstruction algorithm, discuss issues of extended-source flux calibration, and describe CUBISM, a tool that implements these methods for spectral maps obtained with the Spitzer Space Telescope's Infrared Spectrograph.
\end{abstract}

\section{INTRODUCTION}

Spectroscopy has long been at the forefront of astronomical research. From uncovering the elemental abundances underlying the solar spectrum to modern massively parallel surveys probing the scale and structure of the universe, the dispersion of light affords an almost preternatural power of discovery at a distance. The recent advent of large-format optical and infrared detector arrays has revolutionized spectroscopy, enabling many novel methods that capitalize on the growing pixel count. Cross-dispersion of the dispersed beam enables higher spectral resolution and longer slits in compact instruments (e.g., Allington-Smith et al. 1989; Tull et al. 1995; Smith et al. 1998; McLean et al. 1998; Wilson et al. 2001). The use of multiple slits or fibers makes it possible to obtain spectra for hundreds of objects simultaneously over wide fields (e.g., Heacox \& Connes 1992; York et al. 2000; Colless et al. 2001; Le Fèvre et al. 2001; Hook et al. 2003). Integral field spectroscopy of moderately wide areas using image slicing, densely packed fiber bundles, or microlens arrays efficiently maps extended objects (e.g., Tecza et al. 1998; Content 1998; Mandel et al. 2000; Dubbeldam et al. 2000; Bacon et al. 2001; Poglitsch et al. 2006). These methods, along with Fabry-Pérot scanning and other imaging interferometers, fall under the broad category of three-dimensional spectroscopy, which encompasses a set of techniques and instrumental designs that enable the observer to build up spectral cubes with two spatial and one spectral dimension.

\footnotetext{
${ }^{1}$ Steward Observatory, University of Arizona, Tucson, AZ.

${ }^{2}$ Spitzer Science Center, California Institute of Technology, Pasadena, CA.

${ }^{3}$ Department of Physics and Astronomy, University of Wyoming, Laramie, WY.

${ }^{4}$ Max-Planck-Institut für Astronomie, Heidelberg, Germany.

${ }^{5}$ California Institute of Technology, Pasadena, CA.

${ }^{6}$ Institute of Astronomy, Cambridge University, Cambridge, UK.
}

Given finite detector area, a trade-off among field of view, spectral resolution, and instantaneous wavelength coverage must be made for a given three-dimensional spectrograph design. In the limit of zero background, identical throughput and detector performance, and an unchanging point-spread function (PSF), any combination of these three that utilizes the same fractional detector area will offer identical spectral mapping efficiency. Modern integral field spectrographs typically favor larger fields at the cost of resolution or wavelength coverage but have the notable advantage of capturing the entire field at once, which mitigates systematics due to variations in sky background and transmission, and time variations in the PSF (e.g., in adaptive optics applications; Thatte et al. 2000). For observing single unresolved sources, however, integral field spectrographs are relatively inefficient, since they dedicate a large fraction of their detector budget to unused background area. For isolated point sources, single-slit spectroscopy still maintains an advantage. This advantage is even more acute in the ground-based infrared, where, due to the large and variable sky brightness, source and sky must be switched rapidly throughout the observation - on timescales of minutes at near-infrared (NIR) wavelengths $(1-2.5 \mu \mathrm{m})$ and up to $10 \mathrm{~Hz}$ at thermal mid-infrared (MIR) wavelengths $(4-20 \mu \mathrm{m})$. In this regime, single long slits offer another compelling advantage: the ability to chop the source between two positions along the slit, performing in situ sky subtraction with well-characterized slit throughput and minimal loss of observing efficiency.

Unfortunately, single long slits are of more limited use for three-dimensional spectroscopy of extended sources from the ground. In particular, variations in the sky brightness and transmission, as well as the PSF, during the (potentially long) series of integrations required to map out large extended regions pose significant challenges, especially for sources with surface 


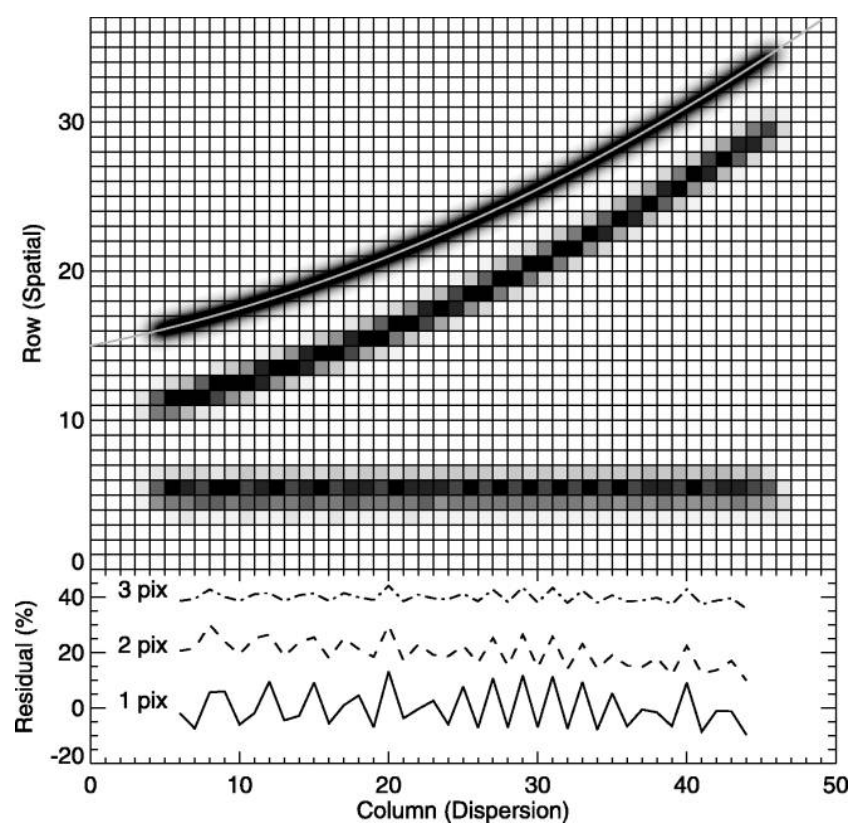

FIG. 1.-Illustration of resampling noise. Top: Top line, an ideal, uniform, curved spectral order, with center indicated by the gray line, is formed from a flat-spectrum source with a Gaussian PSF of constant width FWHM = 1.5 pixels. It is sampled by the pixel elements (middle line) and resampled to straighten the order using linear interpolation, centered precisely on the known order center (bottom line). Bottom: Extractions of three different pixel widths (offset for clarity), demonstrating the purely sample-based noise induced by resampling.

brightness comparable to or below that of the sky. Despite these drawbacks, the scanning method has been used at optical and near-infrared wavelengths to construct moderate-sized maps of bright sources (e.g., Burton \& Allen 1992; Monteiro et al. 2005), and in solar astronomy (e.g., Johanneson et al. 1992), but has been eclipsed in recent years by dedicated integral field techniques. Although they offer high mapping efficiency for extended sources, the reduced efficiency of integral field spectrographs for unresolved sources often dictates that additional long-slit modes be included in instruments that offer these capabilities, increasing complexity.

In space, the various limitations of long slits for conducting three-dimensional spectroscopy over wide fields are minimized, since the astrophysical background is typically smooth and unvarying, and the image characteristics fixed. The Infrared Spectrograph (IRS; Houck et al. 2004) aboard the Spitzer Space Telescope (Werner et al. 2004) includes four sensitive, background-limited spectrograph modules (covering 5-38 $\mu \mathrm{m}$ ) based on fixed single slits, with no moving parts. The Spitzer telescope offers a dedicated spectral mapping mode in which a selected IRS slit is moved in a raster pattern, stopping to obtain one or more exposures at each position, to map out extended regions. Since the IRS is a diffraction-limited spectrograph that delivers a PSF varying in size by roughly a factor of 5 over the full wavelength range, this mode is very useful for obtaining extended-source full-coverage spectrophotometry free from strong aperture bias. Spectral mapping also offers the significant advantage of preserving high efficiency for spectroscopic follow-up of individual point sources while providing the additional capability of very deep, spatially resolved spectroscopy of large, low surface brightness sources, all in a single instrument without moving parts.

Here we describe an approach to reconstructing full spectral cubes from scanned or rastered spectroscopic observations using a single slit. In $\S 2$, we outline a custom algorithm for cube reconstruction, consider issues of extended-source flux calibration in $\S 3$, and finally describe CUBISM, a tool implementing these reconstruction techniques for IRS spectral maps, in $\S 4$.

\section{SPECTRAL CUBE RECONSTRUCTION}

The reconstruction of three-dimensional spectral cubes from a suitably obtained set of overlapping input spectral images (each potentially consisting of multiple spectral orders) differs fundamentally from image mosaicking. The input images carry mixed spatial and spectral information, the relative spatial offsets among the input spectra cannot be readily computed from the mapped data themselves, and ensuring flux conservation requires a dedicated approach. Here we outline the general properties of an algorithm for spectral cube reconstruction, discussing more details of a specific implementation, along with analysis of the constructed cube, in the next section.

\subsection{Resampling Noise}

Modern spectrographs, in particular those which are crossdispersed, rarely form spectral images in which the spatial and spectral axes are aligned with detector rows and columns. Curved orders that cross many detector rows are common. A simple method to deal with these curved order data involves first resampling them, traditionally with a flux-conserving form of linear interpolation, to create a straightened spectral image. After this step, simple summation can be used to extract the final spectrum.

Optimal forms of spectral extraction that employ weighted sums to minimize signal degradation (e.g., Horne 1986) could also be applied to such a straightened image. In practice, however, an unavoidable difficulty enters with this approach when the spatial profile of an unresolved source distributed along the slit is not well sampled. As discussed by Mukai (1990) "resampling noise" results from the initial straightening step, which is retained and amplified by all subsequent processing. Figure 1 illustrates this effect using a single, idealized curved spectrograph order formed from a flat-spectrum source with a constant Gaussian PSF (FWHM $=1.5$ pixels). This continuous profile is then observed with the detector pixel grid (all assumed to have flat and equivalent response) and then straightened by resampling using linear interpolation centered precisely 
on the known order center position. In spectra extracted from the resampled order data, periodic resampling noise of up to $20 \%$ is induced in this example, varying in magnitude with the size of the extraction region. The period depends on the rate at which the order center crosses row boundaries. Although this noise is mitigated at larger extraction sizes, this is no consolation when performing further operations on the resampled spectra, such as estimating the row-by-row slit profile for optimal extraction, as Mukai considered, or remapping the spatially resolved pixels along the slit to a common sky coordinate system, as considered here.

It should be stressed that resampling noise is fully independent from detector or other instrumental properties, or the form of the point-spread function, but is rather an inherent sampling limitation: information on the distribution of flux along the slit has been irrecoverably lost. As an example, consider a PSF that is much smaller than the size of a pixel. In an observed spectral image, you cannot distinguish between the spectrum of a point source having been placed in the center of a pixel or near its edge. If interpolation is performed to shift this spectrum down by, e.g., one-half pixel, in both cases, two equal interpolated pixels result. Real spectrographs present varying and imperfectly determined order positions, both of which can compound this issue. Better sampling alleviates the effect, but it persists at the few percent level even at the typical sampling of 2 pixels per FHWM. In diffraction-limited spectrographs, the PSF sampling can change appreciably from one wavelength end to the other. The IRS spectrograph modules (Houck et al. 2004) were designed to sample the PSF at the long end of each module's wavelength range and thus are significantly undersampled at shorter wavelengths.

To mitigate the effects of resampling noise, it is vital to avoid additional interpolation steps that can introduce it. For the construction of spectral cubes, two opportunities for interpolation can be avoided: initial straightening of the curved order data, and remapping of the rastered set of input spectra onto an output cube grid. Although the information lost by an undersampled spectrum cannot readily be recovered without imposing prior knowledge on the form of the slit profile, avoiding unnecessary interpolation-especially early in the reduction sequence-can minimize the resulting sampling noise. In typical spectral reduction algorithms employing multiple interpolation steps, resampling noise can be reduced by $\sim 10 \%$ using the methods outlined here, strongly dependent on the signal-to-noise ratio of the observations and intrinsic sampling of the spectral instrument.

\subsection{Spatial versus Spectral Degeneracy}

Creating the three-dimensional intensity cube $S(\theta, \phi, \lambda)$ from a series of two-dimensional spectra is fundamentally limited by an implicit degeneracy in the information present in a spectral image. As illustrated in Figure 2, when spectrally mapping with PSF size comparable to or smaller than the slit width, an ambiguity exists in the interpretation of pixels adjacent in the dispersion direction. Given pixel $p_{i j}$ in a single two-dimensional spectral image, which maps to the spectral cube location $S\left(\theta_{k l}, \phi_{k l}, \lambda_{m}\right)$, the adjacent pixel in the dispersion direction $p_{i+1, j}$ could be considered to map either to the same wavelength at adjacent spatial positions within the cube, $S\left(\theta_{k+1, l}, \phi_{k+1, l}, \lambda_{m}\right)$, or to the same spatial position at different wavelengths, $S\left(\theta_{k l}, \phi_{k l}, \lambda_{m+1}\right)$.

In general, both spectral and spatial adjacency are encoded in the slit image. When the PSF is large, very little spatial information remains. When the PSF is small, spatial and spectral information are irrecoverably convolved together within the slit. In the latter case, a single point source with two adjacent spectral lines just blended at the instrument's spectral resolving power will appear identical to the spectrum of a source elongated along the slit width, emitting a single spectral line. Many image-slicing integral field spectrograph designs mitigate (or hide) this problem by sampling the slit width using only a single pixel (e.g., Poglitsch et al. 2004), although without methods to scan wavelength at subresolution, this can adversely impact the reliability of unresolved spectral line measurements. Addressing this degeneracy requires some assumption, with three approaches possible:

1. Assume that no spatial information is available in the slit. Adjacent pixels in the dispersion direction are placed into adjacent wavelength planes in the spectral cube, and all spatial information is provided by the mapping observations.

2. Assume that equal spatial and spectral information is available in the slit. Adjacent pixels in the slit are distributed equally into the spectral cube within individual wavelength planes ("alongside") and at adjacent wavelengths ("beneath").

3 . Assume a varying mix of spatial and spectral information, which can be restated as a mixing angle with respect to planes of constant wavelength, varying from $45^{\circ}$ (equally spatial + spectral) to $90^{\circ}$ (fully spectral).

In this work, we have adopted the first assumption, although others may be advantageous in cases of extreme undersampling of the slit.

\subsection{Remapping the Spectra}

The reconstruction of a full spectral cube $S(\theta, \phi, \lambda)$, with two spatial $(\theta, \phi)$ and one spectral $(\lambda)$ dimension from a set of twodimensional spectral images that have been obtained by mapping a given region of the sky could, in principle, be accomplished in a number of ways. Pixels from the spectra could be remapped onto a cube grid and interpolated. Conversely, pixels in an output cube could be reverse mapped into the original spectral image set, and a weighted average of interpolated values drawn. In order to minimize the effects of resampling noise and to ensure flux conservation, however, a method that avoids unnecessary interpolation steps is preferable.

Such a method is available in direct clipped polygon resam- 


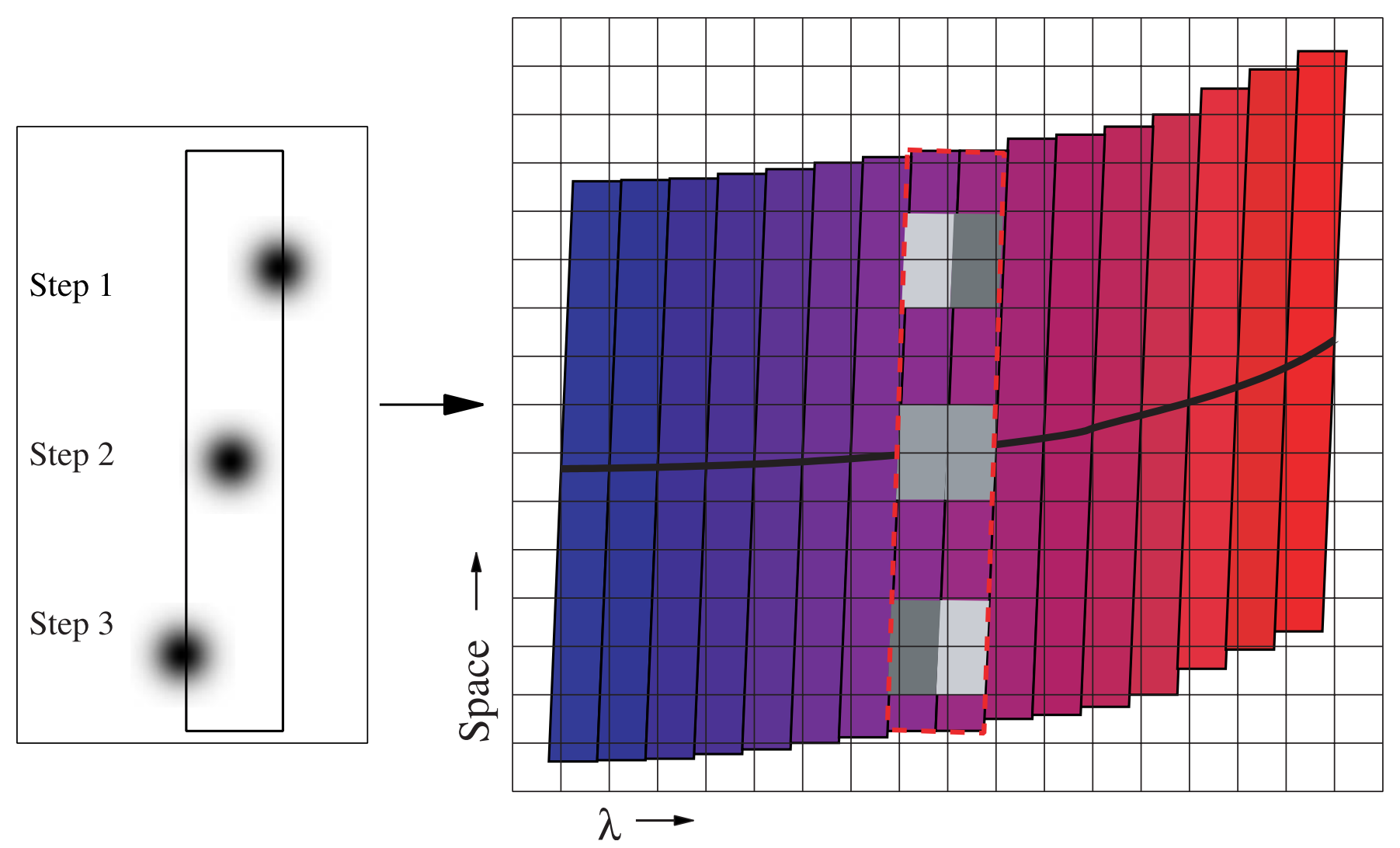

FIG. 2.-Illustration of the spectral/spatial degeneracy in sampled slits. A monochromatic point source with PSF smaller than the slit width of 2 pixels is mapped in three steps perpendicular to the slit (offset along the slit for clarity). In the resulting spectral images, at right, adjacent pixels in the "wavelength" direction can be interpreted as adjacent spatially or spectrally. Single pixel width "pseudorectangles," each of which bounds the contributions from a single wavelength, are shown in color.

pling, in which the contributing weights of individual input pixels are computed by their geometric overlap with an input mask and output grid. Polygon clipping is a mature technique in the field of computer graphics. For the purpose of spectral resampling, simple clipping algorithms (e.g., Sutherland \& Hodgman 1974) suffice.

Since spectral orders are commonly curved and the orientation of the spatial and spectral dimensions can vary at each point along the order, a fundamental element in this algorithm is a means of separating input pixels by wavelength. For this purpose, an abutting set of "pseudorectangles" (PRs), each of which outlines the region of influence of a single wavelength, can be constructed to cover the curved order. The center of each PR is placed at the precise order center, as illustrated in Figure 2, and they may be angled to follow the tilt of spectral lines (common in particular in cross-dispersed spectrograph designs), which itself may vary along the order. In this scheme, an individual input pixel can (and often does) contribute to multiple wavelengths. The exact method of constructing the set of PRs is not critical; as long as the central position along the slit is well determined and matched by the PR centers, there are no gaps in coverage, and the pixel sampling of the under- lying detector device is respected (which in general means that the width of the PR should be 1 pixel).

With the set of PRs in hand, the cube reconstruction algorithm consists of the following steps:

1. Clip each pseudorectangle against the input pixel grid for each of $N$ input spectral images $I$ in a given mapping sequence.

2. Rotate and translate the obtained sets of clipped partial pixel fragments (grouped by image $I$ and wavelength $\lambda$ ) into the output cube grid, according to position and orientation within the map.

3. Clip the offset pixel fragments again against the output (cube) grid.

4. Accumulate the overlap area-weighted sum of contributing pixels in each cube pixel (and, optionally, a parallel uncertainty cube).

The final area-weighted sum can be expressed:

$$
S\left(\theta_{i j}, \phi_{i j}, \lambda_{m}\right)=\frac{\sum_{k=1}^{N} \sum_{x y \in \mathrm{PR}_{m}} \delta_{x y j i m} m_{x y}^{(k)} I_{x y}^{(k)}}{\sum_{k=1}^{N} \sum_{x y \in \mathrm{PR}_{m}} m_{x y}^{(k)} \delta_{x y i j m}},
$$



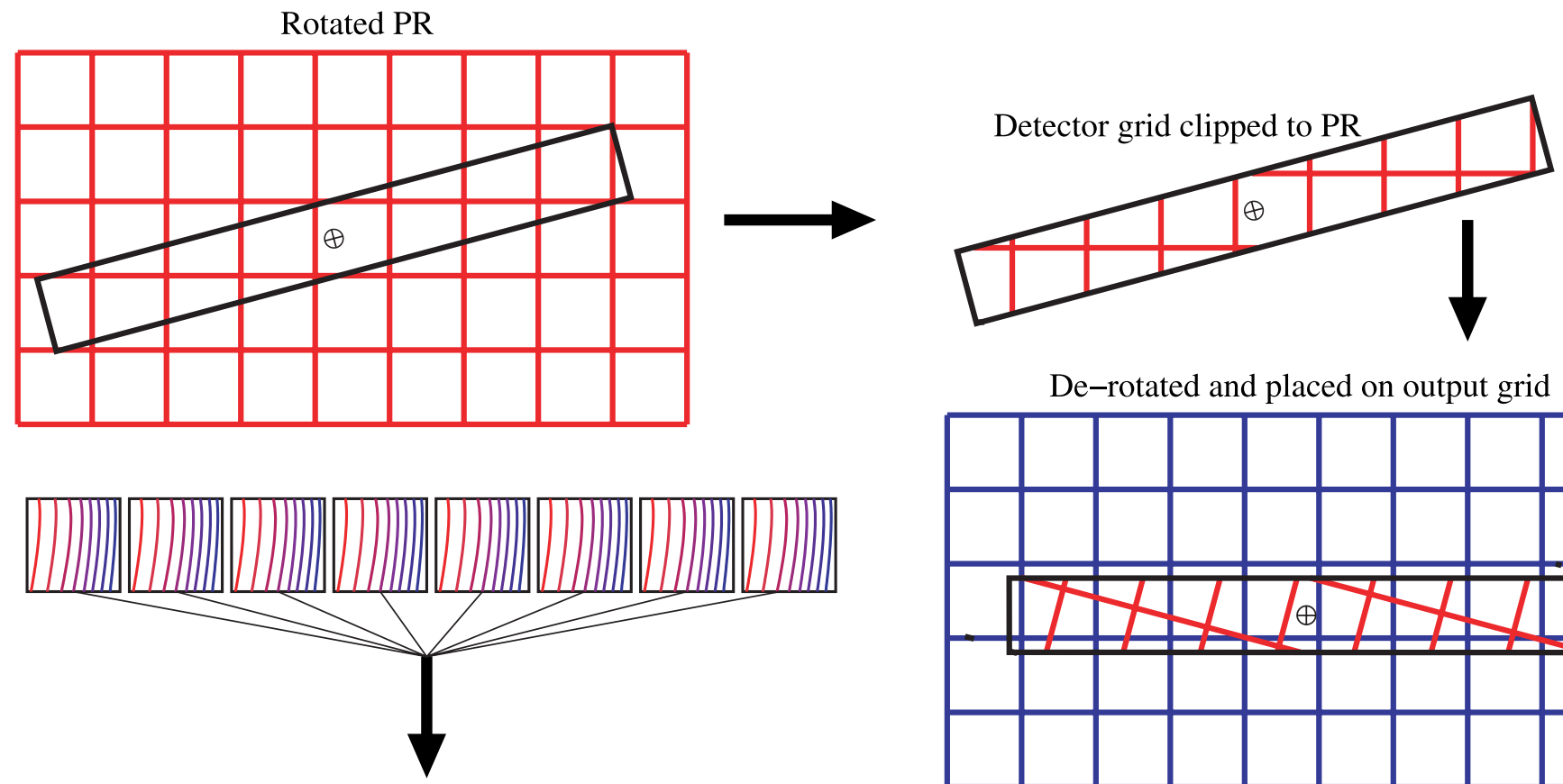

De-rotated and placed on output grid

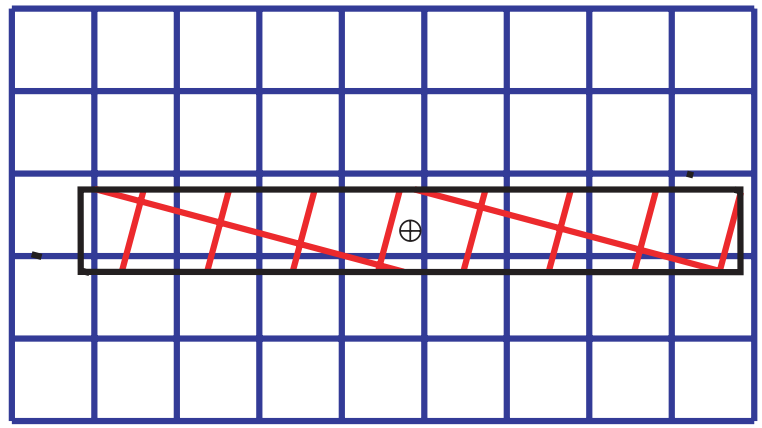

Multiple input spectra contributing
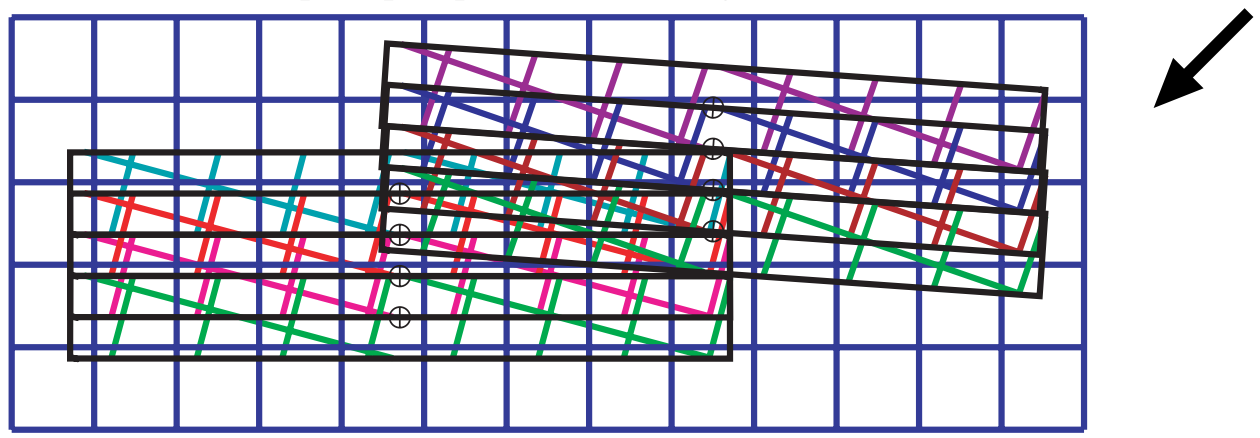

FIG. 3.-Illustration of the two-pass clipping algorithm. From top left: a single pseudorectangle (corresponding to a unique wavelength), shown overlain on the input spectral image ( $r e d$ ) detector grid, is clipped, rotated, and offset into the output cube (blue) pixel grid at the appropriate wavelength plane. Multiple overlapping sets of input spectra are accumulated in the same fashion. A contributing set of input spectra at a slightly different rotation angle is also shown.

where $I_{x y}^{(k)}$ is the data value at pixel $[x, y]$ of the $k$ th input spectral image, $m_{x y}^{(k)}$ is a per image mask used to disable input pixels, and $\delta_{x y j m}$ is the area of overlap in cube pixel $[i, j, m]$ from input pixel $[x, y]$. The overlap area results from two distinct rounds of polygon clipping: (1) clipping the pseudorectangle $m$ (corresponding to wavelength $\lambda_{m}$ ) against the input pixel $[x, y]$ and (2) clipping the resulting "pixel fragment" (which can be a full pixel or a polygonal portion of a pixel), suitably rotated and offset, against the output cube pixel $[i, j]$. A schematic of the two-phase clipping approach is shown in Figure 3.

The core of the cube reconstruction algorithm is the computation of the $\delta$ pixel overlap weights. Their calculation depends on accurate positioning of the pseudorectangles on the input spectrum, including any line tilt at that wavelength, and the absolute position of the slit center (and thus PR center), as well as the rotation of the slit in the output coordinate grid.
Errors in positioning the PR along the order in the input spectra or spatially within the output grid will degrade the achieved spectral and/or spatial resolution, as well as increasing correlation of noise in adjacent pixels. An individual PR may have both an effective rotation induced by instrumental line tilt in the spectral image and a true rotation arising from differential slit position angle at the time of observation with respect to other spectral images in the mapping set. Only the latter should be used to rotate the set of pixel fragments into the output grid (see Fig. 3).

If the spectrograph produces multiple orders from a single slit (e.g., using cross-dispersion), the algorithm of equation (1) can be extended to permit separated groups of input pixels in the wavelength overlap region between orders to contribute to the same output plane. The wavelength sampling of the output cube in the overlap region between orders is chosen to accom- 
modate the maximum spectral resolution present there, and the $\delta$ weighting terms are split linearly between the bracketing output planes, according to their proximity to the output wavelength, e.g., $\delta \rightarrow[f \delta,(1-f) \delta]$, where $f=\left(\lambda_{2}-\lambda_{0}\right) /\left(\lambda_{2}-\right.$ $\left.\lambda_{1}\right)$, for an input wavelength $\lambda_{0}$, and its bracketing output wavelength pair $\left[\lambda_{1}, \lambda_{2}\right]$. This ensures flux conservation even for wavelengths sampled in multiple orders.

If accurate error estimates are available for individual pixels in the input spectral images, they can be propagated through equation (1) using identical weight factors to build a parallel variance cube:

$$
\sigma^{2}\left(\theta_{i j}, \phi_{i j}, \lambda_{m}\right)=\frac{\sum_{k=1}^{N} \sum_{x y \in \mathrm{PR}_{m}} \delta_{x y i j m} m_{x y}^{(k)} V_{x y}^{(k)}}{\sum_{k=1}^{N} \sum_{x y \in \mathrm{PR}_{m}} m_{x y}^{(k)} \delta_{x y i j m}}
$$

where $V_{x y}^{(k)}$ is the variance per input pixel. This algorithm does introduce noise correlations between adjacent pixels (as would interpolative approaches), but in practice these are minimized by employing a high level of pixel redundancy in filled spectral maps, with often a dozen or more input pixels contributing to a single output pixel. Such pixel redundancy occurs naturally for overlapping spectral orders and is greatly increased by constructing mapping observations in which single positions on the sky are sampled by several different parts of the slit (along both its width and length).

The polygon clipping method discussed here bears a superficial similarity to the DRIZZLE algorithm of Fruchter \& Hook (2002) but differs in that it employs exact pixel overlap calculations and two levels of pixel clipping (at the pseudorectangle stage and again into the output cube) and does not shrink pixels or pixel fragments via a "drop factor." Its advantage over related methods to remap curved spectral data is primarily in avoiding unnecessary interpolation steps, which can introduce resampling noise early in the reduction process, as well as enforcing surface brightness conservation implicitly. Another advantage is the ability to track individual contributions from spectral pixels in the source images to the final cube, which can be used readily to reject discrepant pixels.

\subsection{Pixel Rejection and Masking}

The algorithm introduced offers significant statistical power for identifying and rejecting transient or persistent bad pixels. Depending on the sampling redundancy at each pixel within the spectral cube, sigma trimming methods can be used effectively. Since a given input pixel $[x, y]$ will appear many times in a large spectral cube and since each output pixel can have many (dozens or more, depending on map layout) contributing input pixels, statistics can be accumulated to flag outlier pixels. Various schemes can be employed, but a sigma threshold, normalized to an effective measure of the natural spread in the data - the median absolute deviation from the median-proves effective. To identify persistent deviant pixels in all input spectra, it suffices to demand that a given input pixel (at a specific $[x, y])$ be flagged at least some fraction of the times it appears in the output (e.g., 50\%). See $\S 4.5$ for a specific implementation of this approach.

\section{EXTENDED-SOURCE FLUX CALIBRATION}

Accurate flux calibration of extended sources has always proved challenging for slit spectrographs, since uniform, beamfilling astrophysical reference sources of known flux intensity (in $\mathrm{MJy} \mathrm{sr}^{-1}$, for instance) as a function of wavelength are not generally available. Instead, point sources (typically stars) with well-measured or modeled flux distributions are used to provide spectrophotometric reference. The flux calibration of point sources is then, ideally at least, trivial-as long as all science targets are unresolved and are placed in exactly the same position within the slit as the reference calibration sources, all details of beam acceptance profile and diffractive losses, which can vary significantly with wavelength and position along the slit, precisely cancel out, and the ratio of unbiased spectra between target and calibration reference will form an accurate physical flux ratio with the model flux. This ideal situation, although approachable for point sources, is by definition not possible for extended sources, light from which enters the slit at all allowable angles, modulated by the source's angular distribution within the slit. For this reason, some care must be taken to obtain accurate spectrophotometric calibration of extended sources and, in particular, spectral cubes reconstructed from mapping observations of these sources.

There are three main issues with extended-source calibration based on point-source spectrophotometric references, which are discussed in order of their ease of treatment.

\subsection{Aperture Correction}

In imaging point-source photometry, a crucial issue for calibrating extended sources is the aperture over which the pointsource flux is measured, typically stated as a radius in pixels. Assuming that all unresolved sources are measured with the same aperture radius, the derived fluxes do not depend on the aperture size. However, since such an aperture encircles only a fraction of the total flux of a source, an aperture correction must be applied to derive accurate extended-source calibrations from known photometric reference stars (e.g., Reach et al. 2005).

An identical issue occurs in slit spectroscopy, with the extraction aperture width serving the role of the photometric radius. Small aperture widths are desirable for faint sources, since they exclude noisy pixels in the source's profile along the slit. Larger aperture widths, however, minimize aperture corrections, since they capture the bulk of the source flux present in the spectrum. This tension between the desire for larger aperture for unbiased flux measurement and smaller apertures for high signal-to-noise ratio flux recovery has led to PSF fitting methods (e.g., Stetson 1987) and their approximate analog in spectroscopic applications, optimal extraction (e.g., Horne 1986). 
Neither PSF fitting nor optimal extraction is applicable to extended sources, since they rely on the uniform distribution of source flux on the detector or in the slit. The first step of accurate extended-source flux calibration is, therefore, forming an estimate of the aperture losses as a function of wavelength (the "aperture-loss correction function" [ALCF]). This function can be applied to the normally extracted spectrum of spectrophotometric reference stars to estimate the full, undiminished flux at each wavelength in the limit of an infinite aperture. For long slits, the form and magnitude of the ALCF can often be obtained trivially using bright flux reference stars with wide extraction apertures.

\subsection{Slit-Loss Correction}

A slit has finite dimensions and therefore accepts only a portion of the flux of a point source imaged onto it. In diffraction-limited spectrographs, such as the IRS, this slit throughput changes appreciably with wavelength, as the PSF delivered by the telescope grows in size by diffraction. For calibrating point-source spectra, this does not present a difficulty; as long as all point sources, including the flux references, are well centered in the slit, the slit throughput function divides out. For an extended source, however, a calibration system tied to reference point-source observations will necessarily include an implicit correction for the fraction of the point-source flux that the slit admits. In the limit of a perfectly uniform, slitfilling extended source-which can be conceptualized as an infinitely dense grid of point sources with vanishing flux-the diffractive losses out of the slit are exactly offset by the diffractive gains into the slit from sources beyond its geometric boundary.

In this limit, then, a "slit-loss correction function" (SLCF), which is simply the fraction of the flux of a well-centered point source admitted by the slit as a function of wavelength, must be used to correct extended-source fluxes. Without this correction, extended-source fluxes are overestimated. Obviously, the actual magnitude of the true SLCF depends on the observed source flux distribution, which can be intermediate between unresolved and fully uniform. This complicates its use, but in practice, depending on the size of the slit, this detail can often be ignored, and a single correction function can be employed for all extended sources. Estimates of the SLCF can be obtained by convolving models of the PSF (e.g., STinyTim for the Spitzer telescope) with the approximate slit dimensions. An example of such a set of SLCFs for the four spectrograph modules of Spitzer's IRS is shown in Figure 4.

\subsection{Slit Beam Profile}

Although a slit can be formed with precise geometric boundaries, the solid angle it subtends on the sky is broadened due to diffraction. Just as for radio dish measurements, a slit has some beam profile, $B(\theta, \phi)$, which can change with wavelength. Estimating this beam profile function can be very difficult.

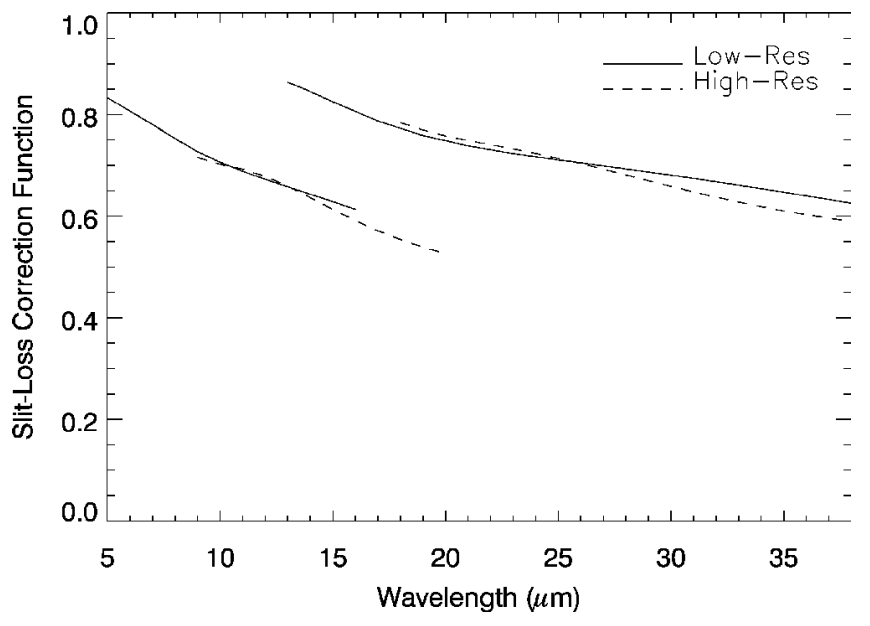

FIG. 4.-Slit-loss correction function for the four IRS modules.

Salama (2000) describe a method for deconvolving the spectrograph beams aboard the Infrared Space Observatory using a series of spectra obtained by rastering a point source around the input slit. Such a method obviously relies heavily on models of the diffraction of the combined telescope and spectrograph optical path but in principle can be used to recover $B$.

When attempting to test the reliability of a given slit-loss correction, for instance by comparing synthetic photometry from the spectra to real photometry over a common wavelength range (e.g., $24 \mu \mathrm{m}$ Spitzer photometry), there is a strong degeneracy with the effective solid angle subtended by the slits, $\Omega_{\text {eff }}=\int B(\theta, \phi) d \theta d \phi$, such that the SLCF can be scaled up or down, with compensating changes to the inferred beam. If the slit is uniform, and unless both corrections are separately known, it is useful to collapse all information of the beam profile to a single number, the effective solid angle per pixel, and derive this number by comparing photometry with spectrophotometry in a sample of extended-source measurements. This effective solid angle per pixel should be approximately equal to the slit width times the pixel scale.

\section{CUBISM}

An implementation of the cube reconstruction algorithm presented in $\S 2$ for spectral mapping observations using Spitzer's IRS spectrograph is available in CUBISM, the Cube Builder for IRS Spectral Mapping. ${ }^{7}$ CUBISM is written in the Interactive Data Language (IDL) and is designed to combine sets of two-dimensional spectral images from IRS mapping observations into single 3D spectral cubes, with two spatial and one spectral dimension. A variety of cube analysis tools are also available, including arbitrary extractions and map creation (e.g., a continuum-subtracted line image). CUB-

\footnotetext{
${ }^{7}$ Other tools are available for the processing of single, staring mode IRS observations-e.g., SMART (Higdon et al. 2004) and SPICE.
} 
ISM, and a manual detailing its use, are available from the Spitzer Science Center. ${ }^{8}$

\subsection{Inputs and Calibration}

CUBISM uses by default the raw two-dimensional basic calibrated data (BCD) spectral images, which have been processed by the IRS pipeline (flat-fielded, corrected for stray light and certain detector anomalies, etc.). Versions of the data without flat-fielding or stray-light correction can optionally be used for testing purposes. It applies the twin clipping reprojection algorithm of $\S 2$, using an input set of calibration data derived primarily from SSC calibration products. A set of pseudorectangles for each order (see $\S 2.3$ ), which delineate the order position and wavelength solution, are constructed directly from the order position and line tilt calibrations. The standard WAVSAMP calibration files provide a similar set of PRs but do not follow the detector's pixel sampling as required to minimize noise correlation and are therefore not employed. Extendedsource SLCF and ALCF flux corrections are applied by default (see $\S 3$ ), the latter by employing a special form of the standard FLUXCON flux calibration input.

The effective solid angle per pixel (the single free parameter in the extended-source flux calibration methods discussed in $\S 3$ ) is calibrated by comparing integrated spectrophotometry over $\sim 1 \operatorname{arcmin}^{2}$ areas to corrected photometry in Multiband Imaging Photometer for Spitzer (MIPS), Infrared Array Camera (IRAC), IRS Blue Peak-up, and ISOCAM data in bright galaxies from the Spitzer Infrared Nearby Galaxies Survey (SINGS) Legacy survey (Kennicutt et al. 2003). In all cases, these solid-angle terms agree with the measured slit width within $10 \%$.

\subsection{Cube Construction}

CUBISM constructs an output cube grid that bounds the mapped region, with default pixel size identical to the sampling in the relevant spectrograph module (ranging from $1.85^{\prime \prime}$ to $5.08^{\prime \prime}$ ). Individual spectra are offset into the output grid according to the requested or reconstructed positions of the slit center during the exposure. The latter, based on contemporaneous telescope attitude reconstruction from gyroscopes and star tracker, yield more stable results. The limited spatial information available in any individual spectral image makes cross-correlation alignment untenable, such that accurate positional accuracy (below the level of the pixel sampling) is crucial for this technique. Resulting positional errors, compared to fixed astrometric systems (e.g., 2MASS), are $\sim 1 "$.

Each of the six subslits-two each for low-resolution modules long-low (LL, 15-37 $\mu \mathrm{m}$ ) and short-low (SL, 5-15 $\mu \mathrm{m}$ ), along with two high-resolution modules short-high ( $\mathrm{SH}, 10$ $20 \mu \mathrm{m})$ and long-high (LH, 20-38 $\mu \mathrm{m})$-are treated indepen-

\footnotetext{
${ }^{8}$ See http://ssc.spitzer.caltech.edu/archanaly/contributed/cubism.
}

dently. A single cube can contain data for only a single subslit, although for the low-resolution modules, it is unimportant how the data were targeted (e.g., the second order of SL can be used from $\mathrm{BCDs}$ in which the first order was targeted, etc.). In the latter case, the frame table that defines absolute positions of all Spitzer apertures is consulted to calculate the position of the offset subslit. Consistent with measurements, spatial distortion along the slits due to projection distortion or other optical effects is assumed negligible. In each of the high-resolution modules, 10 orders are formed by cross-dispersion from the single entrance slit. The cubes are created by merging the order data in the overlap wavelength region, as described in $\S 2.3$.

The fractional contributions of all input pixels contributing to a given output cube pixel are computed and are collectively called the cube "accounts." These can be stored and reread, and they permit high-level statistical methods to be applied to the cube data, for example to exclude outliers (see $\S 4.5$ ). CUBISM was extensively tested on simulated spectral mapping data to validate the algorithm and ensure flux conservation.

For speed, the core clipping functions, which implement a special-purpose reentrant form of the Sutherland-Hodgman polygon clipping algorithm (Sutherland \& Hodgman 1974), are written in $\mathrm{C}$ and autocompiled. If autocompilation fails, an IDLnative version will be used as fallback (at a significant speed penalty).

\subsection{Uncertainty}

Full error cubes are built alongside the data cubes by standard error propagation of equation (1), using, for the input uncertainty $\sigma_{I_{x}}$, the BCD-level uncertainty estimates produced by the IRS pipeline from deviations of the fitted ramp slope fits for each pixel. These uncertainties, which are statistical ramp uncertainties only and neglect other errors in calibration and later processing steps, are used to provide error estimates for extracted spectra and constructed line or continuum maps.

\subsection{Background}

Both to remove the astrophysical foreground and backgrounds (primarily zodiacal and cirrus) and to mitigate the effects of time-varying warm or "rogue" pixels, it is advantageous to subtract near-in-time background frames from each input data record at the 2D level. Suitable background frames (which are free from source contamination in the targeted slit) can be taken from the observations directly (e.g., for smaller targets), from dedicated offset observations, or, if neither is available, from records obtained from the archive at close times (within a few days) and ecliptic latitude $\left(\left|b-b_{0}\right| \lesssim 10^{\circ}\right.$ ). If necessary, additional correction can be made by subtracting a 1D spectrum (e.g., extracted from a dark region within the cube) - equivalent to removing a constant value from each cube plane. 


\subsection{Bad Pixels}

The bad pixel masks, $m_{x y}^{(k)}$ of equation (1), are derived from three sources. Permanently marked nonresponsive pixels from the PMASK are combined with pixels flagged during the exposure for saturation (zero or one ramp sample valid), or flatfield difficulties, which are reported in the BMASK input frames (automatically loaded alongside the BCDs). Additional user bad pixels can be marked either globally (such that pixel $[x, y]$ is disabled in all input spectra) or for individual input records. Both types of bad pixels can be generated automatically, by computing outlier statistics in the accounts information built up during the cube generation (the full record of which input pixels contributed to which output pixels and with what fractional weight). Two parameters, $r_{\sigma}$, the sigma-trim threshold, and $f_{\text {min }}$, the minimum fraction, control automatic bad pixel detection. For each output cube pixel, the contributing pixel residuals (either with or without subtracted background) are tested against an estimate of the contributing pixel deviation $\sigma$ (computed either from a true variance or, for small number contributing, the median absolute deviation from the median value). If a pixel is an outlier, i.e., $\left|I_{x y}-\operatorname{median}\left(I_{x y \in i j m}\right)\right| \geq$ $r_{\sigma} \sigma$, it is tentatively marked bad. If the same pixel is so marked at least a fraction $f_{\min }$ of the times it appears in the cube, it is flagged. Significant power at high spatial frequency (point sources, edges) results in a large natural range of contributing pixel values, such that some care must be taken to ensure that valid data (e.g., strong spectral lines) are not flagged.

\subsection{Components}

The CUBISM interface consists of three main components: the project window, a multipurpose viewer, and a spectrum viewer and map creation interface. Underlying the interface components is a scriptable object-oriented data container, serialized to disk using IDL's own save format (e.g., cube. cpj). All components communicate with each other to present a consistent state. For example, when loading a new set of bad pixels in one component, the displayed bad pixels in another are automatically updated.

\subsubsection{CUBISM Project}

All data, calibration inputs, and cube build settings are collected and operated on from the CUBISM Project pane, shown in Figure 5. From this interface, projects can be loaded and saved, or recovered from disk. Any number of project windows can be opened at a time. Records (individual BCDs) can be added and removed, disabled from the build, combined into background images in various ways, sorted, examined for header information, etc. The cube build parameters and cube build itself are controlled from the project component. The CUBISM project interface also allows bad pixels and background records sets to be loaded, combined, and saved. An individual project targets a single slit in a single module. Sep-

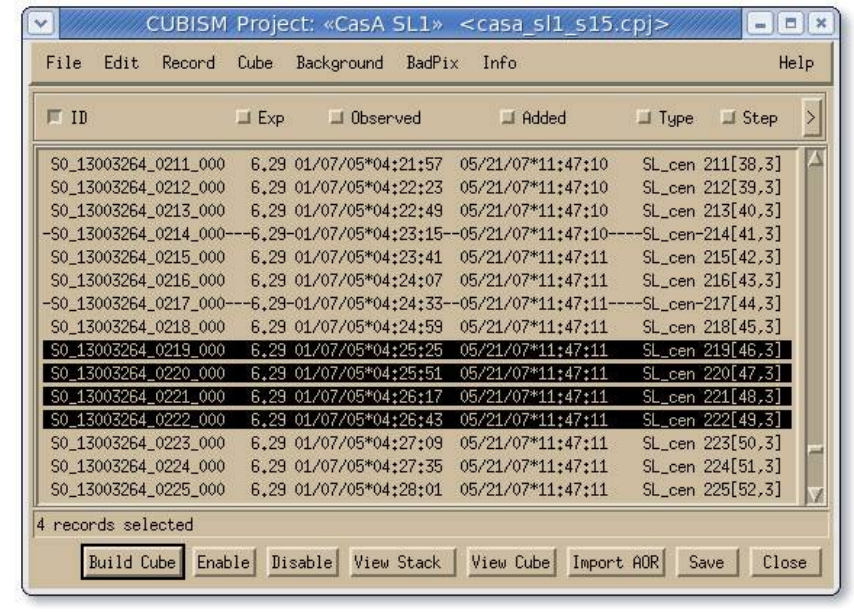

FIG. 5.-CUBISM Project window, with several disabled and selected records.

arate visualization images can be loaded, and feedback is provided on the cube build. Various optimizations are employed to speed up the cube build process, including keeping track of the specific cube pixels affected by changes to bad pixel settings and rebuilding only these. Using the viewer component, records or stacks of records, the assembled cube, or an image loaded for visualizing the position of the slit at each step can be displayed.

\subsubsection{CubeView}

Individual records, stacks of records, spectral cubes or maps, and visualization images can all be viewed in a single viewer interface: CubeView. CubeView has a number of general and special purpose tools that are activated as needed. A single project at a time communicates with one or more instances of the viewer, which modifies itself based on the type of data displayed. For example, if the cube project sends for view an average stack of BCD records, CubeView provides tools for toggling background subtraction, editing the slit-end cutoff positions of the pseudorectangle set, viewing, adding, or removing global and record level bad pixels, etc. When viewing full spectral cubes, an interface to move through the cube planes is provided, and a cube extraction tool is enabled, to construct rectangular extractions or matched region extractions based on preexisting spectra. When viewing cubes, maps, or visualization images, the world coordinate system (WCS) coordinates are reported, whereas with spectral data, the wavelength, order, and any pixel flags are shown. When viewing visualization images, individual records can be selected directly on the overlay (useful for specifying background records). General use tools, including histogram scaling, color map modification, line slice plotting, box statistics, aperture photometry, a pixel table, and others, are always available. 

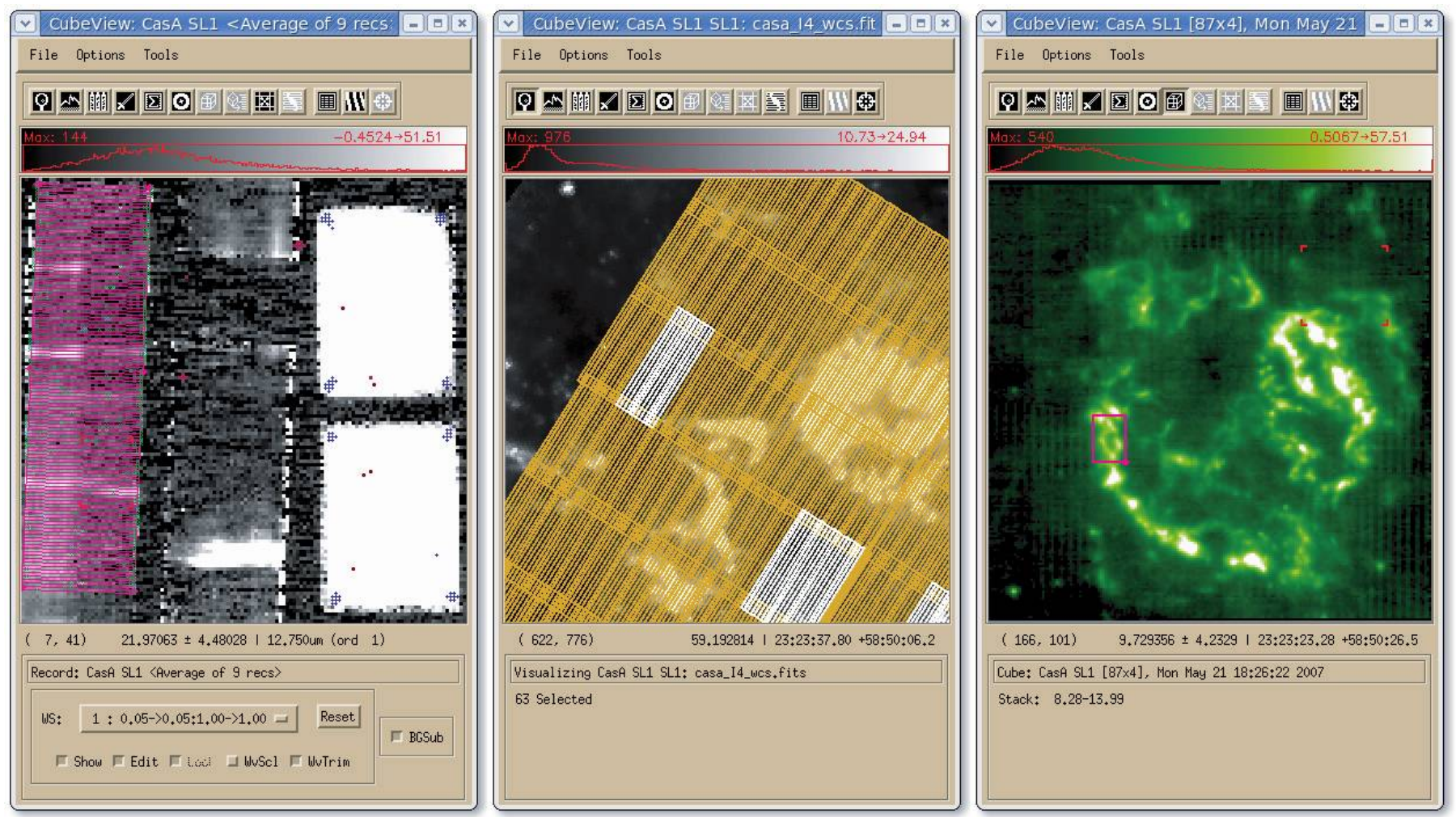

FIG. 6.-CubeView viewer, in three different configurations, showing (from left to right) a stack of BCD records with pseudorectangle set overlaid and bad pixels displayed, a visualization image with the slit positions and selected records shown, and a stack built from the cube itself with an extracted region shown.

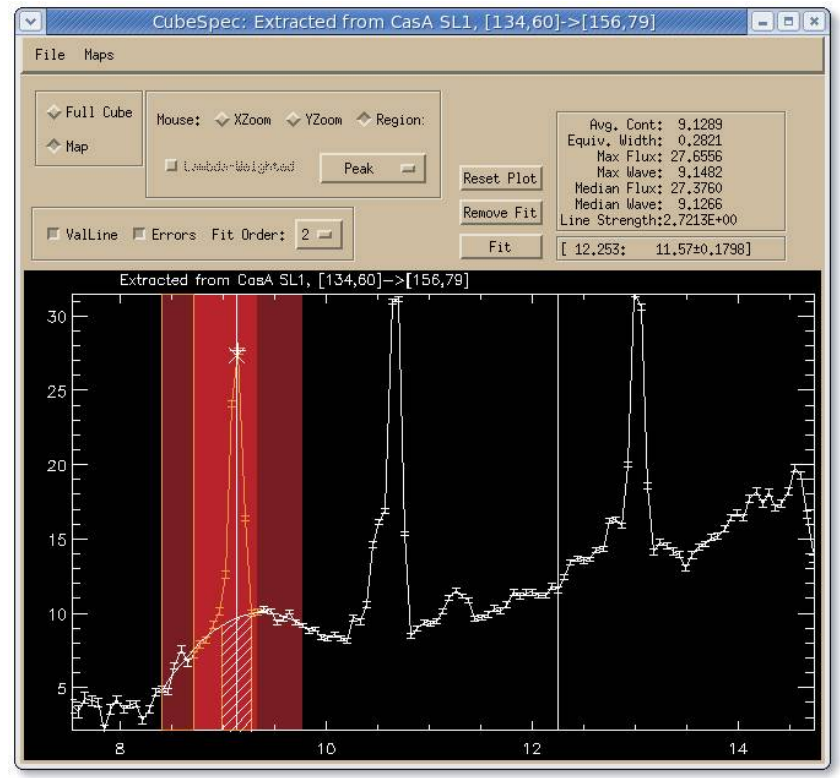

FIG. 7.-CubeSpec window, displaying an extracted spectrum, and map region composed of one peak and two continuum regions. The line has been fitted.
Multiple CubeView instances can be used simultaneously, or a single instance reused to view different types of data. Separate projects loaded in the same session communicate with their own set of CubeView tools. An example of CubeView displaying record spectral data, a visualization image, and map created from a spectral cube is shown in Figure 6.

\subsubsection{CubeSpec}

When an extraction is made from a spectral cube being viewed, the resulting spectrum is displayed in the CubeSpec tool, shown in Figure 7. Map images can be assembled and directly viewed in the associated CubeView viewer by specifying an arbitrary number of foreground and continuum wavelength regions over which to average (e.g., a line region flanked by two continuum regions), with either wavelength-offsetweighted or uniformly weighted averages used to set the continuum in each foreground plane. Map sets can be saved and restored by name, and a custom redshift entered to shift sets (e.g., specified at rest frame) into the observed frame of a target. The tool also provides simple line-fitting capabilities and the ability to create maps using filter curves or other weighting functions (e.g., a $24 \mu \mathrm{m}$ image using the MIPS $24 \mu \mathrm{m}$ filter curve). 


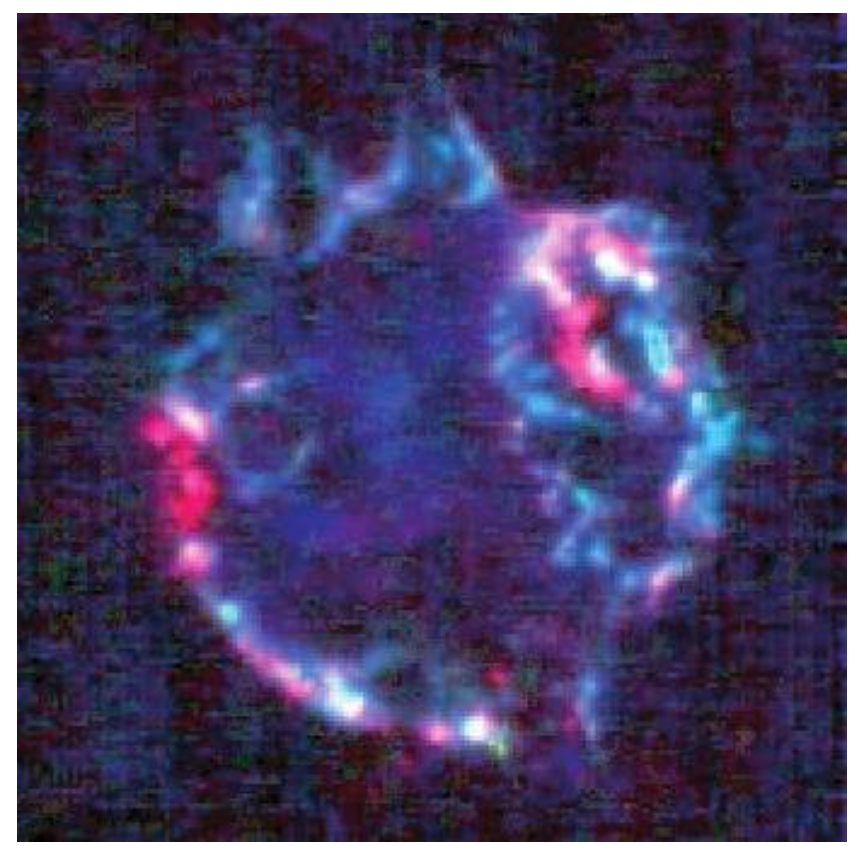

FIG. 8.-Example map created from a SL IRS spectral map (7-15 $\mu \mathrm{m})$ of supernova remnant Cas A, in three continuum-subtracted lines: [Ne II] (red), [Ar III] (green), and [S IV] (blue). Each position in the map has an associated full 5-38 $\mu \mathrm{m}$ spectrum.

\subsection{Outputs}

CUBISM outputs data products in standard FITS and IPAC Table formats. Although the working storage format is IDL SAV file, the full spectral cubes can be exported as FITS, with the third spectral dimension's wavelengths encoded as a lookup table, following the FITS spectral coordinate standards of Greisen et al. (2006). Maps created from the cubes are output as standard 2D FITS images, with information encoding the foreground and background wavelength range(s) used to create them. When uncertainty cubes have been built, accompanying uncertainty FITS files are produced alongside the FITS data products.

Spectral extractions are reproduced in the IPAC Table format (an ASCII format with column delineations, labels, and units) ${ }^{9}$ and include information describing the coordinates of the extraction rectangle. All products have associated WCS coordinate systems attached, with estimated accuracy of 1 ".

\section{OPTIMIZED SPECTRAL MAPPING}

As for deep image mosaics, the redundancy of pixel sampling is the foremost determinant of the quality of a produced spectral cube. Redundancy permits robust statistical rejection of bad pixels, minimizes the impact of variations in slit throughput or

\footnotetext{
${ }^{9}$ See http://irsa.ipac.caltech.edu/applications/DDGEN/Doc/ipac_tbl.html.
}

detector response along the slit, and reduces noise correlation between neighboring output pixels.

In particular for the IRS, with its time-varying rogue pixels, sampling a given position on the sky with at least 4 different detector pixels is crucial for achieving high final cube quality (e.g., using the methods of $\S 4.5$ ). Given the spatial and spectral degeneracy ( $\$ 2.2$ ), it is also critical to step by one-half the slit width in the dispersion direction. Wider step spacing risks gaps or uneven photometric response in the spectral cube, in particular when a random and unknown spatial dither pattern is added to the requested pointing positions. A detailed set of recommendations for planning and executing spectral maps is provided at the SSC. ${ }^{10}$

\section{SUMMARY}

In observing regimes with stable astrophysical background and image characteristics, integral field spectroscopy of extended sources can be achieved efficiently by rastering a single long slit to cover the target area with suitable redundancy. This can significantly reduce instrument complexity and size, in particular if a long-slit mode is already required for efficient follow-up of isolated point sources. These savings in instrument complexity are traded against increased dependence on telescope pointing accuracy — only telescope attitude systems that can track position at angular scales substantially smaller than the smallest slit width provide effective platforms for slit-mapping integral field spectroscopy.

A resampling noise-minimizing, two-pass polygon clipping algorithm for the reconstruction of spectral cubes with two spatial and one spectral dimension from spectral mapping data sets is introduced, and an implementation of this algorithm for Spitzer IRS spectral mapping observations, CUBISM, is described. CUBISM has been used for individual maps comprised of $~ 9000$ individual spectral data frames, for ultradeep blind spectral mapping surveys, and for large area mosaics covering hundreds of square arcminutes. It makes full use of the unparalleled background-limited performance of the IRS spectrograph to provide sensitive integral field infrared spectroscopy. An example custom image created from three continuum-subtracted lines from a large map of the supernova remnant Cassiopeia A is shown in Figure 8.

The authors thank the staff of the IRS instrument support team at the Spitzer Science Center, California Institute of Technology — with special thanks to Phil Appleton, Carl Grillmair, David Shupe, Pat Morris, Sergio Fajardo-Acosta, Patrick Ogle, Jim Ingalls, Harry Teplitz, and Patrick Ogle-for substantial continuing support and advice regarding all aspects of telescope control, instrument calibration, pipeline behavior, and more. We are also indebted to the IRS instrument team at Cornell University_in particular Jim Houck, Vassilis Charmandaris,

\footnotetext{
${ }^{10}$ See http://ssc.spitzer.caltech.edu/irs/documents/specmap_bop.
} 
Jeff Van Cleve, Keven Uchida, Greg Sloan, Sarah Higdon, and Daniel Devost - for much helpful advice, discussions, calibration assistance, and insight into the instrument. Michael Cushing provided helpful discussions regarding aspects of the al- gorithm design. Support for this work, part of the Spitzer Space Telescope Legacy Science Program, was provided by NASA through contract 1224769 issued by JPL/Caltech under contract 1407.

\section{REFERENCES}

Allington-Smith, J. R., et al. 1989, MNRAS, 238, 603

Bacon, R., et al. 2001, MNRAS, 326, 23

Burton, M., \& Allen, D. 1992, Proc. Astron. Soc. Australia, 10, 55

Colless, M., et al. 2001, MNRAS, 328, 1039

Content, R. 1998, Proc. SPIE, 3354, 187

Dubbeldam, M., Content, R., Allington-Smith, J. R., Pokrovski, S., \& Robertson, D. J. 2000, Proc. SPIE, 4008, 1181

Fruchter, A. S., \& Hook, R. N. 2002, PASP, 114, 144

Greisen, E. W., Calabretta, M. R., Valdes, F. G., \& Allen, S. L. 2006, A\&A, 446, 747

Heacox, W. D., \& Connes, P. 1992, A\&A Rev., 3, 169

Higdon, S. J. U., et al. 2004, PASP, 116, 975

Hook, I., et al. 2003, Proc. SPIE, 4841, 1645

Horne, K. 1986, PASP, 98, 609

Houck, J. R., et al. 2004, ApJS, 154, 18

Johanneson, A., Bida, T., Lites, B., \& Scharmer, G. B. 1992, A\&A, 258, 572

Kennicutt, R. C., Jr., et al. 2003, PASP, 115, 928

Le Fèvre, O., et al. 2001, in Deep Fields, Proc. ESO Workshop, ed. S. Cristiani, A. Renzini, \& R. E. Williams (Berlin: Springer), 236 Mandel, H., et al. 2000, Proc. SPIE, 4008, 767

McLean, I. S., et al. 1998, Proc. SPIE, 3354, 566
Monteiro, H., Schwarz, H. E., Gruenwald, R., Guenthner, K., \& Heathcote, S. R. 2005, ApJ, 620, 321

Mukai, K. 1990, PASP, 102, 183

Poglitsch, A., et al. 2004, Proc. SPIE, 5487, 425 2006, Proc. SPIE, 6265, 425

Reach, W. T., et al. 2005, PASP, 117, 978

Salama, A. 2000, in ISO Beyond Point Sources: Studies of Extended Infrared Emission, ed. R. J. Laureijs, K. Leech, \& M. F. Kessler (ESA SP-455; Noordwijk: ESA), 7

Smith, J. D. T., et al. 1998, Proc. SPIE, 3354, 798

Stetson, P. B. 1987, PASP, 99, 191

Sutherland, I. E., \& Hodgman, G. W. 1974, Commun. Assoc. Comput. Machinery, 17, 32

Tecza, M., Thatte, N. A., Krabbe, A., \& Tacconi-Garman, L. E. 1998, Proc. SPIE, 3354, 394

Thatte, N., et al. 2000, in ASP Conf. Ser. 195, Imaging the Universe in Three Dimensions, ed. W. van Breugel \& J. Bland-Hawthorn (San Francisco: ASP), 206

Tull, R. G., MacQueen, P. J., Sneden, C., \& Lambert, D. L. 1995, PASP, 107, 251

Werner, M. W., et al. 2004, ApJS, 154, 1

Wilson, J. C., et al. 2001, PASP, 113, 227

York, D. G., et al. 2000, AJ, 120, 1579 